\title{
Automation Tools for Finite Element Analysis of Adhesively Bonded Joints
}

\author{
Farhad Tahmasebi, Ph.D. \\ NASA-Goddard Space Flight Center (GSFC) \\ Mechanical Jystems Analysis \& Simulation Branch - Code 542 \\ Greenbelt, MD 20771 - USA \\ farhad.tahmasebi@gsfc.nasa.gov
}

\section{ABSTRACT}

This article presents two new au:omation tools that obtain stresses and strains (sheas and peel) in adhesively bonded joints. For a given adhesively bonded joint Finite Element model, in which the adhesive is characterised using springs, these automation tools read the corresponding input and output files, use the spring forces and deformations to obtain the adhesive stresses and strains, sort the strisses and strains in descending order, and generate plot files for $3 \mathrm{D}$ visualisation of the stress and strain fields. Grids (nodes) and elements can be numbered in any order that is convenient for the user. Using the automation tools, trade-off studies, which are needed for design of adhesively bonded joints, can be performed very quickly.

Keywords: Finite Element Analysis, Adhesively Bonded Joints, Automation, Stiffness, Shear/Peel Stress/Strain

\section{NOMENCLATURE}

All of the symbols used in this article are defined in Table 1.

Table 1: Definition of symbols.

\begin{tabular}{|c|c|}
\hline Symbol & Definition \\
\hline$A$ & area of plat elements \\
& used to mods l adherends \\
$E$ & adhesive elasticity modulus \\
$f_{x, i}$ & X spring force at grid $i$ \\
$f_{y, i}$ & Y spring for a at grid $i$ \\
$f_{z, i}$ & Z spring forse at grid $i$ \\
$G$ & adhesive shear modulus \\
$i$ & grid number \\
\hline
\end{tabular}

Table 1: Definition of symbols (continued).

\begin{tabular}{|c|c|}
\hline Symbol & Definition \\
\hline$K_{p}$ & peel spring stiffness \\
$K_{s}$ & shear spring stiffness \\
$P$ & peel force \\
$V$ & shear force \\
$\Delta$ & peel deflection \\
$\delta$ & shear deformation \\
$\delta_{x, i}$ & X spring deformation at grid $i$ \\
$\delta_{y, i}$ & Y spring deformation at grid $i$ \\
$\delta_{z, i}$ & Z spring deformation at grid $i$ \\
$\epsilon$ & adhesive peel strain \\
$\epsilon_{i}$ & peel strain at grid $i$ \\
$\gamma$ & adhesive shear strain \\
$\gamma_{i}$ & shear strain at grid $i$ \\
$\eta$ & thickness of adhesive \\
$\nu$ & adhesive Poisson's ratio \\
$\sigma$ & adhesive peel stress \\
$\sigma_{i}$ & peel stress at grid $i$ \\
$\tau$ & adhesive shear stress \\
$\tau_{i}$ & shear stress at grid $i$ \\
\hline
\end{tabular}

\section{INTRODUCTION}

L.J. Hart-Smith has performed a lot of pioneering work in analysis and design of adhesively bonded joints (e.g., [1], [2]).

Many others have used Finite Element methods and springs (linear or non-linear) to model adhesives in bonded joints (e.g., [3]). The Finite Element model of a typical adhesively bonded joint is shown in Figure 1. If forces and/or moments are applied to such a joint, spring forces and deformations can be used to determine the stresses and strains in the adhesive. 
This document describes two F(IRTRAN programs

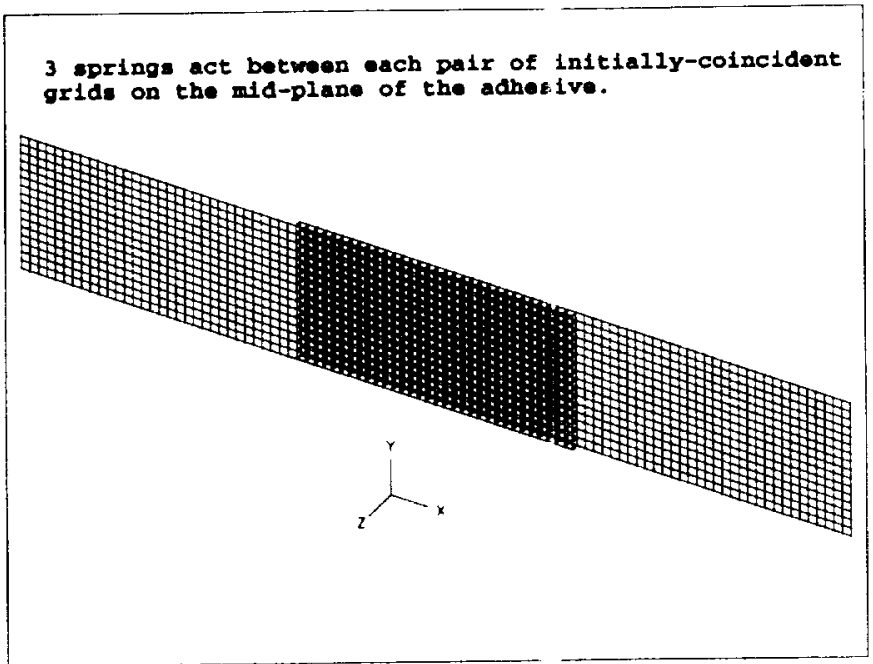

Figure 1: Finite Element model of a bonded joint.

which automate the process of ol taining stresses and strains in adhesively bonded joints. Given an adhesively bonded joint model, these programs read the corresponding NASTRAN (NAS A Structural Analysis, a Finite Element software par:kage widely used in the Aerospace industry) input and output files, use the spring forces or deformations to obtain the adhesive stresses or strains at the mid-plane grid points, sort the stresses and strains in descending order, and generate Mathematica (a popular symbolic mathematics and graphics software package) plot files for $3 \mathrm{D}$ visualisation of the stress an 1 strain fields.

Note that the grids and springs can be numbered in any order. The prourams determine the pairs of grids which are initially-coincident, and the spring triplets which correspond to such grid pairs. In the earlier attempts at NASA -GSFC to model adhesives in bonded joints, the anilyst had to number the grids and/or springs in specilic orders so that the $\mathrm{XYZ}$ spring triplets acting between pairs of initiallycoincident adhesive grids could be identified. Such ordering of grids and/or spring, can be very timeconsuming.

The spring stiffness values are determined from the adhesive stress-strain curve and the bonded joint geometry (see Section 4).

\section{THE SPRING MODELING PROCESS}

The modeling process involves the following steps:

1. A fine mesh of grids is created in the mid-plane of the bonded joint.

2. For every grid in the mid-plane mesh, an initially-coincident grid is created.

3. Three springs are placed between each pair of initially-coincident grids. These springs act in the $\mathrm{X}, \mathrm{Y}$, and $\mathrm{Z}$ directions; where the $\mathrm{X}$ and $Y$ axes are parallel to the overlap plane of the bonded joint, and the $\mathrm{Z}$ axis is defined by the right-hand-rule.

4. Rigid elements are used to connect the midplane grids to the corner grids of the plate elements which represent the adherends (see Figure 2).

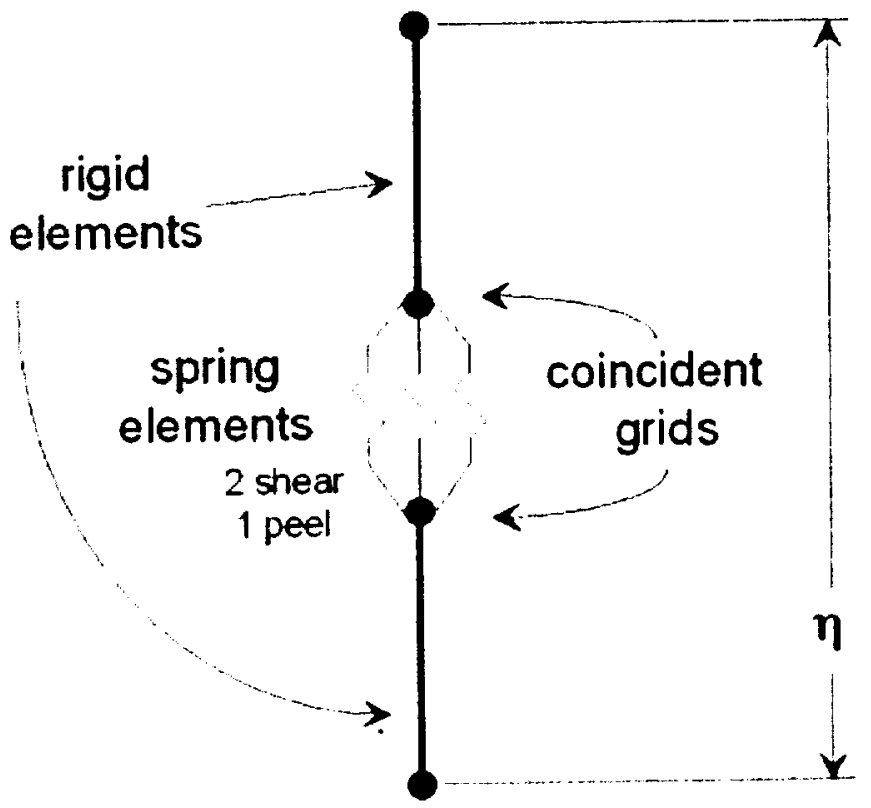

Figure 2: Depiction of shear and peel springs.

\section{DETERMINATION OF THE SPRING STIFFNESS VALUES}

\subsection{Stiffness of Shear Springs}

To obtain the shear spring stiffness values, we can use the following general relationships:

$$
\tau=G \gamma, \quad \tau=\frac{V}{A}, \quad \delta=\gamma \eta
$$


Symbols in equation (1) are defined in Table 1. Therefore,

$$
\frac{V}{A}=G \frac{\delta}{\eta}
$$

We also know that:

$$
V=K_{s} \delta
$$

where $K_{s}$ is defined in Table 1 . Combining equations (2) and (3), we get

$$
K_{s}=\frac{G A}{\eta}
$$

Equation (4) gives the shear sping stiffness values as a function of the adhesive sheir modulus and the model geometry, and can be used in assigning stiffness values to the shear springs of an adhesive Finite Element Model. Note that if quadrilateral shell elements are used to model the adhierends (a common practice), then:

$$
A=A_{\text {internal }}=2 A_{\text {edge }}=4 A_{\text {corner }}
$$

where $A_{\text {internal }}, A_{\text {edge }}$, and $A_{\text {corn:r }}$ represent the element areas to be used in equation (4) for calculating the shear spring stiffness values.

\subsection{Stiffness of Peel Springs}

To obtain the peel spring stiffnes: values, we can use the following general relationshiys:

$$
\sigma=E \epsilon, \quad \sigma=\frac{P}{A}, \quad,=\frac{\Delta}{\eta}
$$

Symbols in equation (6) are defined in Table 1. Therefore,

$$
\frac{P}{A}=E \frac{\Delta}{\eta}
$$

We also know that:

$$
P=K_{p} \Delta
$$

where $K_{p}$ is defined in Table 1 . Combining equations (7) and (8), we get

$$
K_{p}=\frac{E A}{\eta}
$$

Equation (9) gives the peel spring stiffness values as a function of the adhesive elasticity modulus and the model geometry, and can be used in assigning stiffness values to the peel springs of an adhesive Finite Element Model. Note that if quadrilateral shell elements are used to model the adherends (a common practice), then the relationships specified in equation (5) apply, and must be used in calculating the peel spring stiffness values.

If the adhesive elasticity module is not published in the manufacturer's literature, it can be calculated from its Poisson's ratio and its shear modulus using the following equation:

$$
E=2 G(1+\nu)
$$

Loss and Keyward [3] discuss variations of equation (10) for various stress states.

\section{DESCRIPTION OF THE AD- HESIVE STRESS PROGRAM}

Given a bonded joint model, the program executes the following operations.

1. Prompts the user for the name of NASTRAN input and output files.

2. Locates and stores all of the spring ID's, the corresponding grid ID's, and the corresponding grid coordinates in the NASTRAN input file.

3. Identifies the pairs of grids which are coincident in the unloaded model.

4. Identifies the spring triplets ( $\mathrm{X}, \mathrm{Y}$, and $\mathrm{Z}$ ) for the pairs of initially-coincident grids.

5. Locates and stores the spring forces in the corresponding NASTRAN output file.

6. Assigns the stored spring forces to the appropriate springs in the triplets identified in step 4.

7. Determines adhesive shear and peel stresses at the mid-plane grids using the following equations.

$$
\begin{gathered}
\tau_{i}=\frac{\sqrt{f_{x, i}^{2}+f_{y, i}^{2}}}{A} \\
\sigma_{i}=\frac{f_{z, i}}{A}
\end{gathered}
$$

The symbols in equations (11) and (12) are defined in Table 1.

8. Sorts the shear and peel stresses in descending order and writes them to an output file.

9. Writes the coordinates of the mid-plane grid points and their corresponding shear and peel stresses to plot files. 
The plot files mentioned in step 9 are used to generate 3D representations of the slear and peel stress fields using Mathematica. Figure 3 shows the 3D shear stress field for the joint represented in Figure 1. To efficiently execute the operations spec-

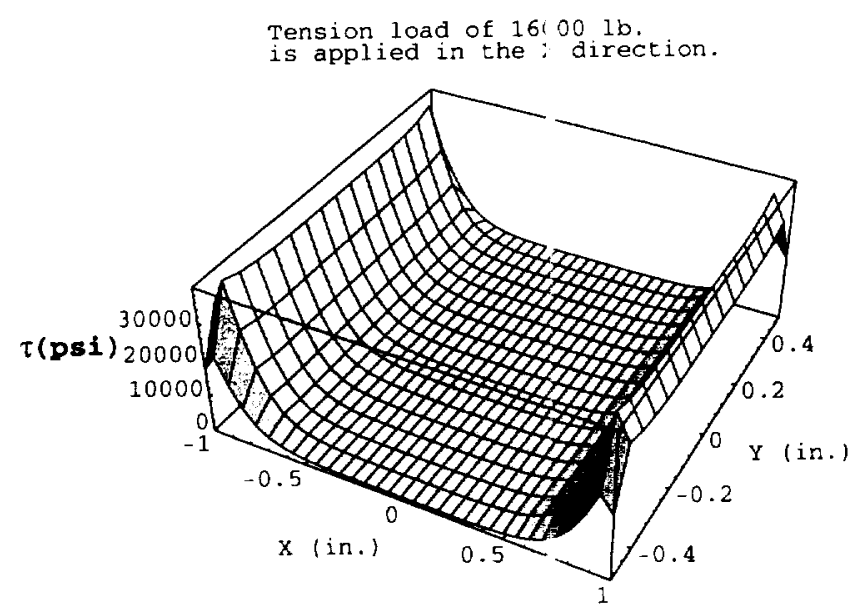

Figure 3: Shear stress in a bonded joint.

ified in steps 3,4 , and 6 ; the program invokes various sorting subroutines. These sorting operations and other details of the prograin can be found in the source code (adhsv_strs.f) which is located at the http://femci.gsfc.nasa.gov/adhes ve site.

\section{DESCRIPTION OF THE AD- HESIVE STRAIN PROGRAM}

Equations (11) and (12) show that the adhesive shear and peel stresses are dependent on the area of the plate elements used to represent the adherends. For complicated joints, it may not $b$ ? possible to have a uniform area for such plate elements. Therefore, as an alternative, a program was witten to determine shear and peel strains which are independent of the plate element area, and dependent on the adhesive thickness which is normally unif,$r m$.

For a given bonded joint model, the program starts by executing steps 1 through 4 that the Adhesive Stress program goes through (sre the previous section). Next, the program performs the following operations.

1. Locates and stores the mid-plane grid point displacements in the NASTRAN output file.
2. Calculates and stores the adhesive spring deformations from the grid point displacements obtained in the previous step.

3. Assigns the stored spring deformations to the appropriate elements in the spring triplets corresponding to the pairs of initially-coincident grids (see step 4 of the previous section).

4. Calculates adhesive shear and normal strains at the mid-plane grids using the following equations.

$$
\begin{gathered}
\gamma_{i}=\frac{\sqrt{\delta_{x, i}^{2}+\delta_{y, i}^{2}}}{\eta} \\
\epsilon_{i}=\frac{\delta_{z, i}}{\eta}
\end{gathered}
$$

The symbols in equations (13) and (14) are defined in Table 1.

5. Sorts the shear and normal strains in descending order and writes them to an output file.

6. Writes the coordinates of the mid-plane grid points and their corresponding shear and normal strains to plot files.

The shear and normal strain plot files are read into Mathematica to generate 3D representation of the strain fields. Figure 4 shows the 3D shear strain field for the bonded joint represented in Figure 1. The program invokes various sort-

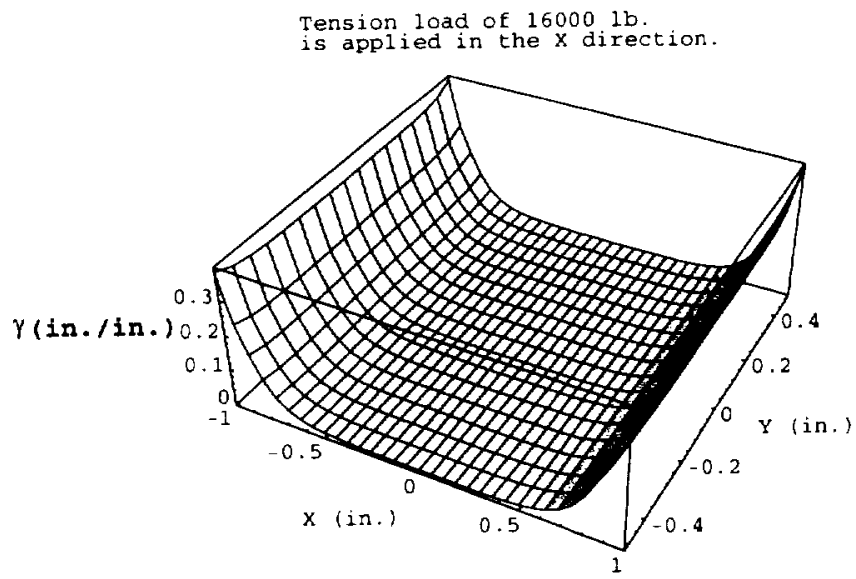

Figure 4: Shear strain in a bonded joint.

ing subroutines to efficiently execute some of the steps specified above. These sorting operations and other details of the program can be found in the source code (adhsv_strn.f) which is located at the http://femci.gsfc.nasa.gov/adhesive site. 


\section{SAMPLE OUTPU'T FILES}

\subsection{Adhesive Stress Program Output}

A short portion of a sample output file for the adhesive stress program is shown below.

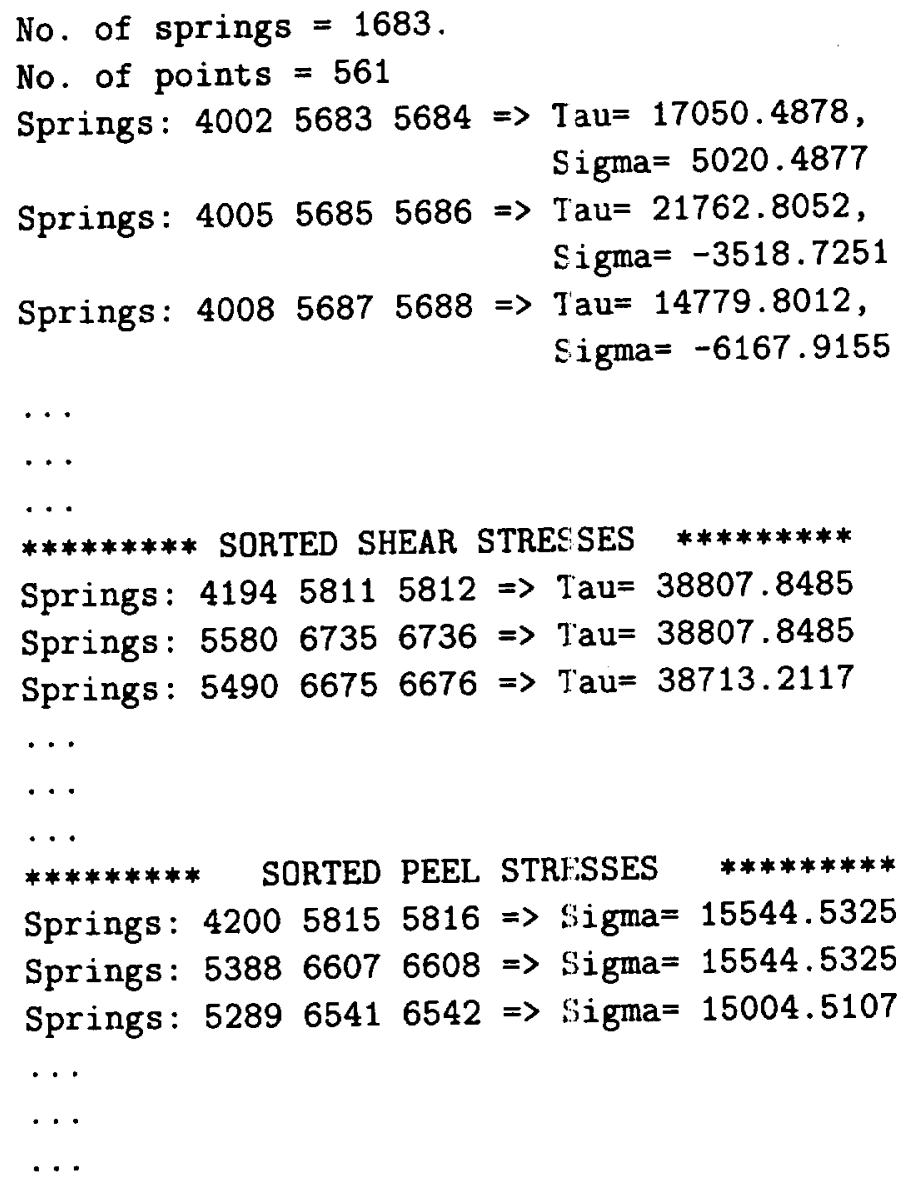

\subsection{Adhesive Strain Program Output}

A short portion of a sample output file for the adhesive strain program is shown belıw.

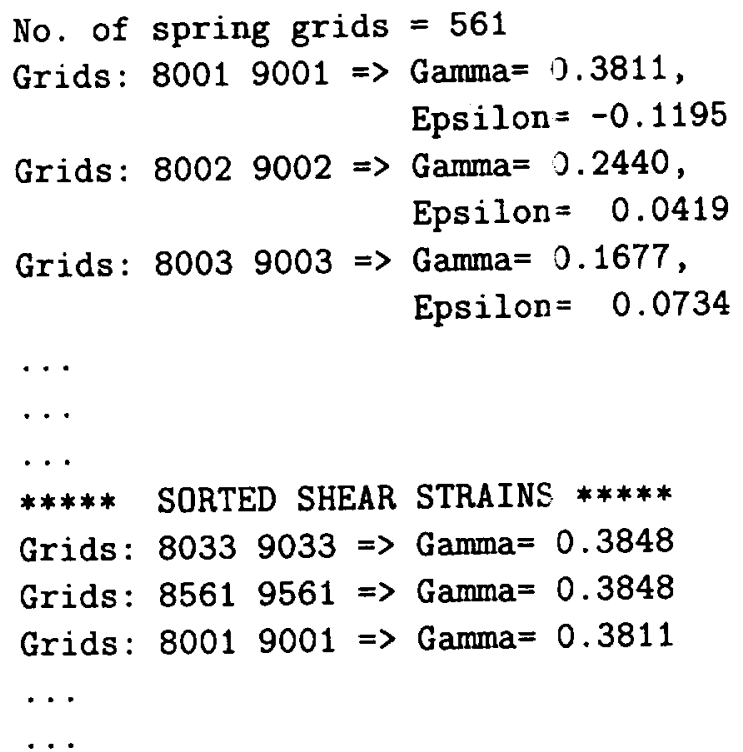

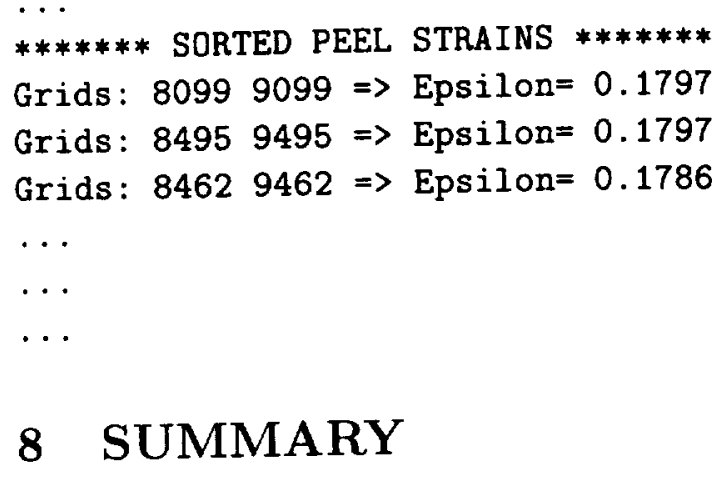

\section{SUMMARY}

In this article, two new automation tools are introduced and described.

The automation tools are used to quickly determine shear and peel stresses and strains in adhesively bonded joints. As a result, trade-off studies for design of such joints can be performed rapidly.

Given an adhesively bonded joint NASTRAN model, in which the adhesive is characterised using springs, the two automation tools read the corresponding input and output files, use the spring forces and deformations to obtain the adhesive stresses and strains, sort the stresses and strains in descending order, and generate plot files for 3D visualisation of the stress and strain fields.

The automation tools can handle any scheme used by the user to number the model elements and grids (nodes).

The results of both programs have been verified by hand-calculating stresses and strains at various adhesive grid points.

\section{REFERENCES}

[1] Hart-Smith,L.J., Adhesive-Bonded Single-Lap Joints,NASA CR-112236, Douglas Aircraft Company, 1973.

[2] Hart-Smith,L.J., Advances in the Analysis and Design of Adhesive-Bonded Joints in Composite Aerospace Structures, Proceedings of 19th National SAMPE Symposium and Exhibition, Society for the Advancement of Material and Process Engineering, 1974, p. 722-737.

[3] Loss, K.R., and Keyward, K.T., Modeling and Analysis of Peel and Shear Stresses in Adhesively Bonded Joints, AIAA paper 84-0913, 1984. 